\title{
Design and control of a parallel linkage wrist for robotic microsurgery
}

\section{Citation}

Degirmenci, Alperen, Frank L. Hammond III, Joshua B. Gafford, Conor J. Walsh, Robert J. Wood, Robert D. Howe. 2015. Design and Control of a Parallel Linkage Wrist for Robotic Microsurgery. In Proceedings of the IEEE/RSJ International Conference on Intelligent Robots and Systems (IROS), Hamburg, Germany, September 28-October 2, 2015: 222-228. doi: 10.1109/ IROS.2015.7353378.

\section{Published Version}

doi:10.1109/iros.2015.7353378

\section{Permanent link}

http://nrs.harvard.edu/urn-3:HUL.InstRepos:25659061

\section{Terms of Use}

This article was downloaded from Harvard University's DASH repository, and is made available under the terms and conditions applicable to Open Access Policy Articles, as set forth at http:// nrs.harvard.edu/urn-3:HUL.InstRepos:dash.current.terms-of-use\#OAP

\section{Share Your Story}

The Harvard community has made this article openly available.

Please share how this access benefits you. Submit a story.

Accessibility 


\title{
Design and Control of a Parallel Linkage Wrist for Robotic Microsurgery
}

\author{
Alperen Degirmenci, Student Member, IEEE, Frank L. Hammond III, Member, IEEE, \\ Joshua B. Gafford, Student Member, IEEE, Conor J. Walsh, Member, IEEE, \\ Robert J. Wood, Member, IEEE, Robert D. Howe, Fellow, IEEE
}

\begin{abstract}
This paper presents the design and control of a teleoperated robotic system for dexterous micromanipulation tasks at the meso-scale, specifically open microsurgery. Robotic open microsurgery is an unexplored yet potentially a high impact area of surgical robotics. Microsurgical operations, such as microanastomosis of blood vessels and reattachment of nerve fibers, require high levels of manual dexterity and accuracy that surpass human capabilities. A 3-DoF robotic wrist is designed and built based on a spherical five-bar mechanism. The wrist is attached to a 3-axis commercial off-the-shelf linear stage, achieving a fully dexterous system. Design requirements are determined using motion data collected during a simulated microanastomosis operation. The wrist design is optimized to maximize workspace and manipulability. The system is teleoperated using a haptic device, and has the required bandwidth to replicate microsurgical motions. The system was successfully used in a micromanipulation task to stack $1 \mathrm{~mm}$-diameter metal spheres. The micromanipulation system presented here may improve surgical outcomes during open microsurgery by offering better accuracy and dexterity to surgeons.
\end{abstract}

\section{INTRODUCTION}

Microsurgical operations are performed throughout the body to perform reconstruction, reattachment, and reanimation of tissue. These operations, such as microanastomosis of blood vessels and reattachment of nerve fibers, require high levels of manual dexterity and accuracy that surpass human capabilities. For instance, in vitroretinal surgery, a $10 \mu \mathrm{m}$ accuracy is desired [1]; however even trained surgeons have a root mean square (RMS) tremor between $49-133 \mu \mathrm{m}$ at the tip of their surgical tool [2].

Researchers have been working on building robot-assisted microsurgery (RAMS) systems that can provide the necessary positioning precision for such operations, however these novel systems are not suited to dexterous teleoperated open microsurgery. The SteadyHand platform from Johns Hopkins [3], and the Intra-Ocular Dexterity Robot (IODR)

This work was supported by the Harvard University School of Engineering and Applied Sciences, and the Wyss Institute for Biologically Inspired Engineering. Any opinions, findings, and conclusions or recommendations expressed in this material are those of the authors and do not necessarily reflect the views of the Wyss Institute.

A. Degirmenci, J.B. Gafford, C.J. Walsh, R.J. Wood, and R.D. Howe are with the School of Engineering and Applied Sciences, Harvard University, Cambridge, MA 02138 USA. F.L. Hammond III is with the Department of Mechanical Engineering, Massachusetts Institute of Technology, Cambridge, MA 02139 USA. R.D. Howe is also with the Harvard - MIT Division of Health Sciences \& Technology, Cambridge, MA 02139 USA. E-mail: \{adegirmenci, jgafford, walsh, rjwood, howe\} eseas.harvard.edu, fhammondemit.edu.

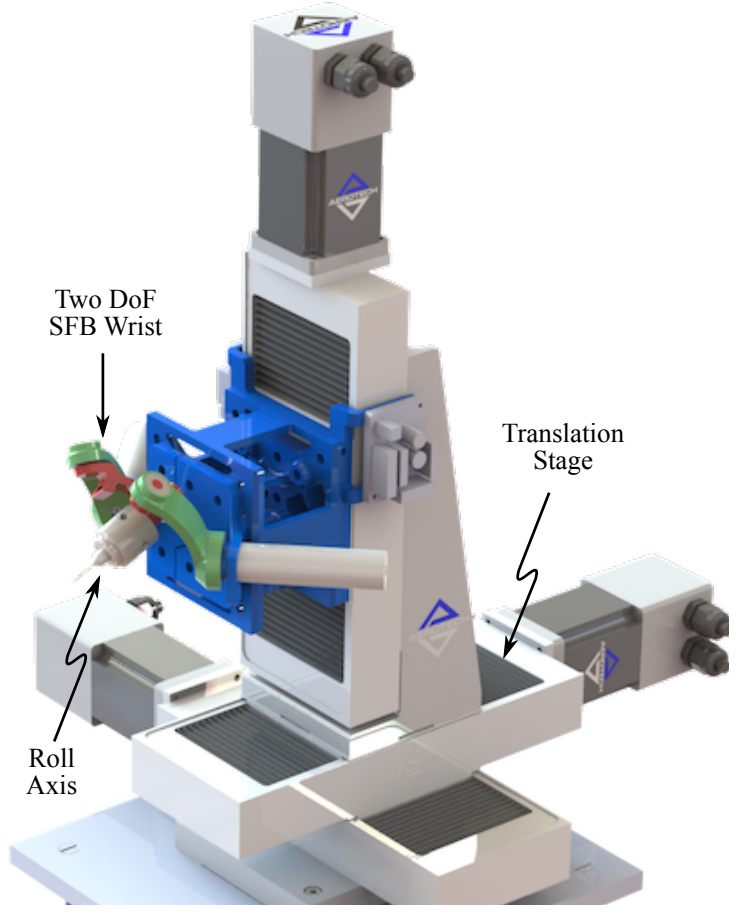

Fig. 1. CAD model of the dexterous manipulation setup. The wrist design is based on the 2-DoF spherical five-bar linkage mechanism, with an additional roll DoF.

from Columbia [4] focus on vitroretinal surgery. The Micron system from Carnegie Mellon aims to cancel out hand tremor using a handheld device [5]. The UBC MotionScaling Teleoperation System (MSTS) is a force reflecting microsurgery system teleoperated in a multi-stage macromicro fashion [6]. The RAMS Workstation from JPL was aimed at microsurgery however the system acted more as a third-arm and could not actually hold and operate a microsurgical needle [7]. A two arm, four degree of freedom (DoF) robotic system from SRI International was used to perform arterial anastomosis in rats, but the operation time was two to three times as long as that in conventional microsurgery [8]. Robotic open microsurgery is an unexplored yet potentially a high impact area of surgical robotics. Several researchers have used the da Vinci Surgical System from Intuitive Surgical, Inc. to perform microanastomosis operations [9][11], however the da Vinci system is intended for macroscale laparoscopic surgery, and lacks the appropriate tools for microsurgery.

This paper explains the design and control of a novel 6DoF robotic system for use in microsurgical operations. The 
system comprises a 3-DoF XYZ positioning stage, and a 2DoF spherical five-bar (SFB) parallel linkage robotic wrist (2-RR) with a third roll DoF that is actuated by a miniature motor that is located coaxially with the output shaft. Commercial off-the-shelf, cost-effective linear stages with submicron-level accuracy are used for positioning (Fig. 1). In Section II we derive the kinematic relations, present our work on optimizing the workspace and manipulability of the system, and explain the system architecture and control methods. Section III describes system characterization methods and an analysis of the experimental results; and Section IV presents conclusions and future work. To the authors' knowledge, this is the first teleoperated robotic microsurgery system to use an SFB wrist. The system presented here will be a valuable tool in studying the characteristics of surgeon's motions during microsurgery, understanding teleoperation at the meso-scale, and may one day become a valuable tool in the operating room.

\section{SYSTEM DESIGN AND CONTROL}

The following design specifications and criteria were considered when determining the requirements for the new system: the number of DoF, workspace, resolution, mechanism type, inertia, stiffness, speed, force, bandwidth, and control type [12].

\section{A. Surgical Motion Characterization}

In order to determine design specifications, data was collected during a simulated microanastomosis operation. This task was deemed appropriate for this study, because microanastomosis is considered by surgeons as one of the most difficult procedures to perform due to the challenges of manipulating small, wet, delicate, and highly compliant vascular tissue. An expert surgeon's tool position, orientation, and acceleration, as well as the force imparted on the phantom tissue were recorded during the experiment. This data helped quantify workspace requirements, motion

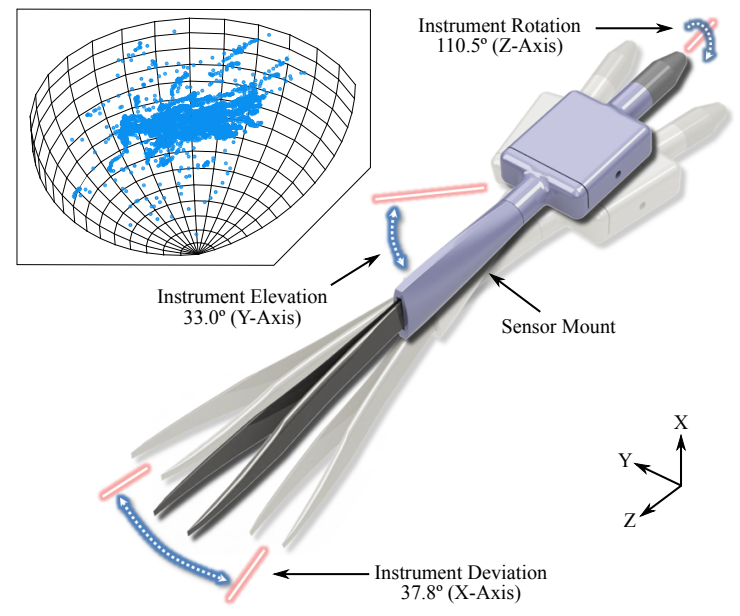

Fig. 2. Figure illustrating the surgical tool instrumentation for a pair of tweezers, and the ranges of angular motion measured during the surgical motion characterization experiment. Inset shows the orientation data projected onto the surface of a sphere. characteristics, and the force profile during the experiments [13].

Surgical tools (forceps, tweezers, and needle drivers) were fitted with accelerometers (ADXL 335, Analog Devices Inc.) and electromagnetic (EM) trackers (trakSTAR, Ascension Technologies Inc.) to record tool acceleration and pose. The EM trackers have a spatial resolution of $0.5 \mathrm{~mm}$ and angular resolution of $0.1^{\circ}$, and the accelerometers have a range of $\pm 3 \mathrm{~g}$ of acceleration. A force measurement plate, fitted with a high-precision 6-axis force-torque transducer (Nano17 6axis transducer, ATI Industrial Automation, Inc.), was used to measure forces imparted by the surgeon on the phantom tissue. The Nano17 can measure forces with resolutions as low as $3.125 \mathrm{mN}$ and moments of $0.0156 \mathrm{mNm}$. Lacrimal duct tubing of $1 \mathrm{~mm}$ diameter was used as a blood vessel phantom. Four interrupted sutures were placed in each mock vessel. The simulated procedure (one vessel anastomosis) took roughly 15 minutes. The maximum force sensed was $82.9 \mathrm{mN}$. The angular motion bandwidth was $0.0-1.34 \mathrm{~Hz}$. The tool was rotated a maximum of $110.5^{\circ}$ in axial rotation, $33.0^{\circ}$ in elevation, and $37.8^{\circ}$ in lateral deviation (Fig. 2). The translational workspace used by the surgeon was $85.9 \mathrm{~mm} \times$ $110.0 \mathrm{~mm} \times 44.8 \mathrm{~mm}$. Inset in Fig. 2 shows a plot of the surgeon's tool orientation represented by the blue dots.

\section{B. Mechanism Type}

Serial chain manipulators are widely used in robotic systems due to their well-understood kinematics; however they have an inherent tradeoff between positioning accuracy and system bandwidth. Higher positioning accuracy requires higher stiffness, which increases inertia, however higher inertia reduces system bandwidth. Parallel manipulators, on the other hand, do not suffer from this tradeoff since the actuators can be grounded while still directly driving the joints. Thus parallel mechanisms have increased system bandwidth and transparency due to the reduced distal inertia, while also maintaining high precision. Parallel structures are also capable of supporting and exerting higher loads and forces. One disadvantage of parallel mechanisms is that they

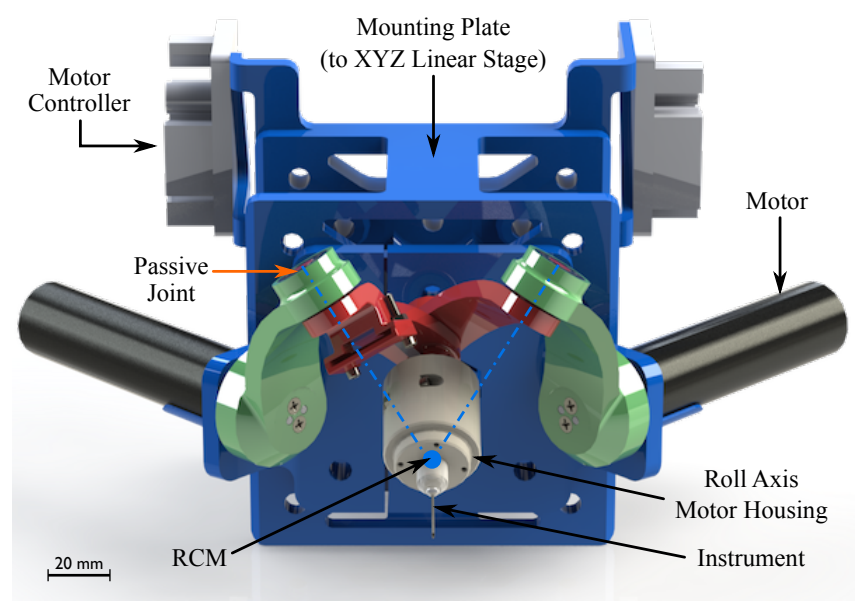

Fig. 3. A CAD rendering of the wrist design. Instrument elevation and lateral deviation are provided by the spherical five-bar mechanism, while rotation is handled by an $\varnothing 8 \mathrm{~mm}$ motor that is coaxial with the instrument. 
TABLE I

A COMPARISON OF VARIOUS ROBOTIC MICROSURGICAL SYSTEMS.

\begin{tabular}{|c|c|c|c|}
\hline System & Target Application & Mechanism Type & Active DoFs \\
\hline \hline SteadyHand [3] & Vitroretinal & XYZ Stage + Roll + Slider-crank & 5 \\
\hline RAMS [7] & Eye & Serial revolute joints & 6 \\
\hline Micron [5] & Tremor reduction & Parallel linkage (3-RㅍS) & 3 \\
\hline IODR [4] & Vitroretinal & Stewart Platform + Flexible End-Effector & $6+2$ \\
\hline Our System & Trauma \& Plastic & XYZ Stage + SFB (2-RRR) + Roll & 6 \\
\hline
\end{tabular}

generally have smaller workspaces. Humans make use of parallel structures in manipulation tasks as well. During a 'precision grasp' [14] (e.g. while writing with a pen, or holding tweezers), two or three fingers are used to grasp and manipulate the object, forming a parallel linkage. Table I gives a list of mechanism types and the number of degrees of freedom for various microsurgical systems.

In this design, a hybrid approach was taken in order to exploit the simplicity and large workspace of serial manipulators combined with the precision and lower distal inertia of parallel structures. For XYZ translation, linear translation stages were used for their simplicity, large workspace, and cost-effectiveness. For orientation, a wrist was designed based on the spherical five-bar (SFB) parallel linkage mechanism (Fig. 3). This parallel mechanism is chosen for its good precision, low inertia, and high stiffness. The use of a SFB for robotic surgery has been proposed before in [4], [15]-[17], but not in the context of microsurgery.

The SFB has five revolute joints, the axes of which coincide at a single point. This point is the center of a sphere, and all five of the linkages lie on the surface of this sphere. This point is a mechanical remote center of motion (RCM) where changes in orientation do not cause a change in position. The SFB has a limited range of motion, and the workspace has singularities. However, for this application, the surgical data indicates that the SFB should have the required workspace, while also delivering the benefits of parallel mechanisms.

Most minimally-invasive surgery (MIS) robots have an RCM, either implemented mechanically and/or in software (i.e. virtual RCM), in order to eliminate lateral forces on the tissue surrounding the entry port. However in open microsurgery there is no entry port, therefore the choice of RCM location is arbitrary. The most sensible option is to make the tip of the end-effector act as an RCM, which results in changes in orientation causing zero change in translation at the tooltip. In this case, minimizing the distance between the

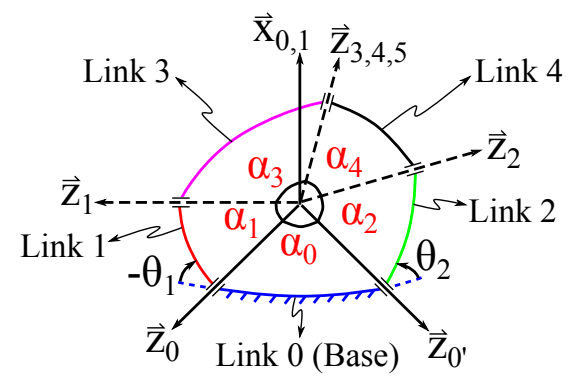

Fig. 4. Nomenclature for kinematics derivations. tooltip (i.e. virtual RCM) and the mechanical RCM would be advantageous because this minimizes the control effort in the XYZ stages required to cancel out the translation of the tooltip due to orientation changes.

\section{Kinematics}

For parallel mechanisms, the inverse kinematics problem is easier to solve than the forward kinematics problem. A number of researchers have worked on solving the kinematics problem for parallel manipulators. Our derivation of the kinematics of the spherical five-bar wrist follows the methodology employed in [17], [18].

TABLE II

D-H PARAMETERS FOR THE SFB.

\begin{tabular}{c||ccccc}
\hline Link & $0 \rightarrow 1$ & $1 \rightarrow 3$ & $0 \rightarrow 0^{\prime}$ & $0^{\prime} \rightarrow 2$ & $2 \rightarrow 4$ \\
\hline$\alpha_{\mathbf{i}}$ & $\alpha_{1}$ & $\alpha_{3}$ & $\alpha_{0}$ & $\alpha_{2}$ & $\alpha_{4}$ \\
\hline$\theta_{\mathbf{i}}$ & $\theta_{1}$ & $\theta_{3}$ & 0 & $\theta_{2}$ & $\theta_{4}$ \\
\hline
\end{tabular}

Fig. 4 shows the conventions followed in these derivations. Frame 0 is defined as the base frame. The two arms of the SFB can be treated as two separate serial chains: $0 \rightarrow 1 \rightarrow 3$, and $0 \rightarrow 0^{\prime} \rightarrow 2 \rightarrow 4$. Then, the rotation matrices that describe the orientation of the end-effector in base coordinates can be written as

$$
R_{3}^{0}=R_{1}^{0} R_{3}^{1}, \quad \text { and } \quad R_{4}^{0}=R_{0^{\prime}}^{0} R_{2}^{0^{\prime}} R_{4}^{2} .
$$

In order to ensure loop closure, two constraints are used which apply to the axes of rotation where the two kinematic chains are coupled, defined as

$$
R_{3_{z}}^{0} \cdot R_{4_{z}}^{0}=1, \quad \text { and } \quad R_{3_{z}}^{0} \cdot R_{4_{x}}^{0}=0
$$

First constraint in Eq. 2 specifies that the $z$ component of the rotation matrices should be collinear, while the second constraint asserts that the $x$ component should be normal to the $z$ component. The rotation matrices for each chain can be found in [18].

Let $\mathbf{u}$ be a unit vector that points along $\vec{z}_{5}$. $\mathbf{u}$ can be represented in base coordinates as

$$
\begin{gathered}
\mathbf{u}^{\mathbf{0}}=\left[\begin{array}{l}
u_{x} \\
u_{y} \\
u_{z}
\end{array}\right]=R_{3}^{0}\left[\begin{array}{l}
0 \\
0 \\
1
\end{array}\right]=R_{3_{z}}^{0} \\
\mathbf{u}^{\mathbf{0}^{\prime}}=\left[\begin{array}{l}
u_{x}^{\prime} \\
u_{y}^{\prime} \\
u_{z}^{\prime}
\end{array}\right]=\left[\begin{array}{ccc}
1 & 0 & 0 \\
0 & c_{\alpha_{0}} & s_{\alpha_{0}} \\
0 & -s_{\alpha_{0}} & c_{\alpha_{0}}
\end{array}\right]\left[\begin{array}{l}
u_{x} \\
u_{y} \\
u_{z}
\end{array}\right]=R_{4_{z}}^{0^{\prime}}
\end{gathered}
$$


where $s_{\theta}$ and $c_{\theta}$ are $\sin \theta$ and $\cos \theta$. From Eq. 3 and 4 we can write

$$
c_{\theta_{3}}=\frac{c_{\alpha_{1}} c_{\alpha_{3}}-u_{z}}{s_{\alpha_{1}} s_{\alpha_{3}}} \quad \text { and } \quad c_{\theta_{4}}=\frac{c_{\alpha_{2}} c_{\alpha_{4}}-u_{z}^{\prime}}{s_{\alpha_{2}} s_{\alpha_{4}}} .
$$

Now the angles $\theta_{3}$ and $\theta_{4}$ can be calculated as

$$
\theta_{3,4}=\operatorname{atan} 2\left( \pm \sqrt{1-c_{\theta_{3,4}}^{2}}, c_{\theta_{3,4}}\right)
$$

with $\theta_{3}$ and $\theta_{4}$ known, $\theta_{1}$ and $\theta_{2}$ can now be calculated. Eq. 8 can be decomposed as

$$
\left[\begin{array}{l}
u_{x} \\
u_{y}
\end{array}\right]=\left[\begin{array}{cc}
c_{\theta_{1}} & s_{\theta_{1}} \\
-s_{\theta_{1}} & c_{\theta_{1}}
\end{array}\right]\left[\begin{array}{c}
s_{\alpha_{3}} s_{\theta_{3}} \\
s_{\alpha_{1}} c_{\alpha_{3}}+c_{\alpha_{1}} s_{\alpha_{3}} c_{\theta_{3}}
\end{array}\right]
$$

from which an expression for angle $\theta_{1}$ is finally obtained as

$$
\theta_{1}=\operatorname{atan} 2\left(-u_{y} \psi_{1}+u_{x} \psi_{2}, u_{x} \psi_{1}+u_{y} \psi_{2}\right)
$$

where $\psi_{1}=s_{\alpha_{3}} s_{\theta_{3}}$ and $\psi_{2}=s_{\alpha_{1}} c_{\alpha_{3}}+c_{\alpha_{1}} s_{\alpha_{3}} c_{\theta_{3}}$. An expression for $\theta_{2}$ can be derived in the same manner. Note that the inverse kinematics problem has four solutions: two solutions for both $\theta_{3}$ and $\theta_{4}$, corresponding to the 'elbow up' and 'elbow down' cases.

The Jacobian maps joint velocities to end-effector velocities. The Jacobian for the SFB can be defined as

$$
\left[\begin{array}{c}
\omega_{x} \\
\omega_{y} \\
\omega_{z}
\end{array}\right]=\mathbf{J}\left[\begin{array}{l}
\dot{\theta}_{1} \\
\dot{\theta}_{2}
\end{array}\right] \text {. }
$$

Following the methodology from [19], we compute the Jacobian as

$$
\mathbf{J}=\left[\begin{array}{ll}
\frac{\vec{z}_{1} \times \vec{z}_{5}}{\left(\vec{z}_{0} \times \vec{z}_{1}\right) \cdot \vec{z}_{5}} & \frac{\vec{z}_{2} \times \vec{z}_{5}}{\left(\vec{z}_{0^{\prime}} \times \vec{z}_{2}\right) \cdot \vec{z}_{5}}
\end{array}\right] .
$$

\section{Mechanism Isotropy and Optimization}

For any given actuator with some angular accuracy (typically set by encoder resolution), the end-effector accuracy will vary throughout the workspace. It is important to limit this variability in the usable workspace, i.e. to increase the isotropy of the device, in order to have a system that behaves consistently. The condition number of the Jacobian matrix is generally used as the metric for isotropy, defined as

$$
\kappa(\mathbf{J})=\|\mathbf{J}\|\left\|\mathbf{J}^{-1}\right\|, \kappa \in \mathbb{R}:[1, \infty)
$$

where $\|\cdot\|$ is the weighted Frobenius norm such that

$$
\|\mathbf{J}\|=\sqrt{\operatorname{tr}\left(\mathbf{J W J}^{T}\right)}
$$

where $\mathbf{W}=\left[\begin{array}{cc}1 / 2 & 0 \\ 0 & 1 / 2\end{array}\right]$ is a weighting matrix [20], [21]. Let $\xi=1 / \kappa$ such that $\xi \in \mathbb{R}:[0,1]$, which behaves better in computations.

A mechanism with $\xi=1$ at all configurations is an isotropic mechanism. Parallel mechanisms are notorious for their anisotropy, where the ratio between input and output changes dramatically throughout the workspace. Having $\xi>0.5$ throughout the usable workspace should lead to a well-behaved system with a more uniform velocity profile and accuracy across the workspace [21].

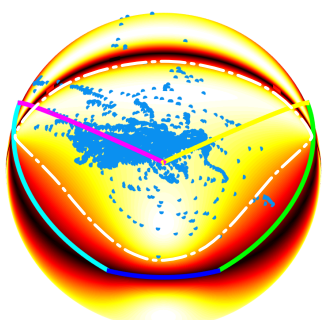

$40^{\circ}, 85^{\circ}, 85^{\circ}$

WGCI: 0.49

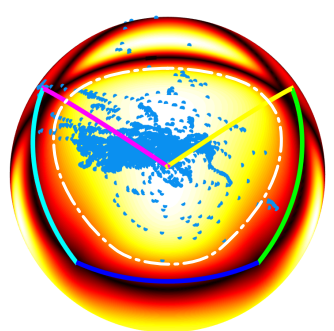

$70^{\circ}, 70^{\circ}, 70^{\circ}$

WGCI: 0.48

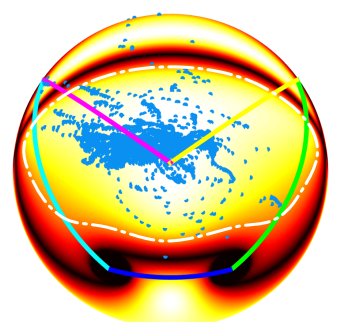

$45^{\circ}, 85^{\circ}, 76.7^{\circ}$

WGCI: 0.57

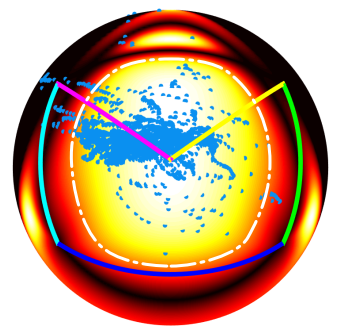

$90^{\circ}, 60^{\circ}, 60^{\circ}$

WGCI: 0.52

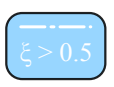

Fig. 5. A number of link angle combinations and their corresponding conditioning index plots. Brighter colors indicate a better conditioning index. Dotted black lines delineate the dexterous workspace regions, where $\xi>$ 0.5. Anastomosis data (shown in light blue) is superimposed on the SFB workspace to indicate the desired workspace envelope. The five circular arcs represent the linkages of the mechanism, with the base linkage illustrated in dark blue. Compared to other designs, the optimized design (top right) has the highest WGCI, and contains the most number of anastomosis points in its dexterous workspace.

The global conditioning index (GCI), $\eta$, was proposed in [22] as a measure of the global behavior of the manipulator condition number, defined as

$$
\eta=\frac{\int_{W} \xi d W}{\int_{W} d W}
$$

where $W$ is the workspace of the manipulator (not to be confused with the weighting matrix $\mathbf{W}$ from Eq. 12). The integrals in this equation are often approximated by some finite sampling method applied to the workspace, since computing the integral analytically is not feasible.

For the SFB optimization, we use a modified form of the GCI as the loss function. We first subdivide the workspace, which lies on the surface of a sphere, into a grid. For each cell $i$ in the grid, $\xi_{i}$ is computed. Each $\xi_{i}$ in the manipulator workspace is assigned a weight based on the density of surgical motion (Fig. 2) at that orientation. We express this weighted global conditioning index (WGCI) as

$$
\eta=\frac{1}{N} \sum_{i=0}^{W} \xi_{i} n_{i}
$$

where $\xi_{i}$ is the condition index at the patch of workspace that is sampled, $n_{i}$ is the number of data points (from the mock microanastomosis experiment) contained in this patch, and $N$ is the total number of data points from the experiment, plus the number of patches.

A constrained optimization was performed with the constraints $\alpha_{1}=\alpha_{2}, \alpha_{3}=\alpha_{4}$ to maintain mechanism symmetry, 
and $\pi / 4 \leq \alpha_{i} \leq \pi / 2$. The workspace was subdivided into a $60 \times 60$ grid (only one quadrant of the workspace was taken into account, since the majority of the surgical motion data fits in this quadrant). Depending on the initial conditions, the optimization converged to two optimal designs with similar WGCI (Table III). Fig. 5 shows conditioning index plots for these two designs, and two non-optimal designs. Even though the design on the bottom right $(\mathrm{WGCI}=0.52)$ has a smaller workspace where $\xi \geq 0.5$, it still has a higher WGCI, indicating that its condition index map aligns better with the anastomosis data.

The workspace for the SFB mechanism can be measured in terms of the area spanned on the surface of a unit sphere, expressed in terms of the azimuth (lateral deviation) and elevation change permitted by the mechanism. The optimized design has a workspace with $72^{\circ}$ in elevation and $144^{\circ}$ in lateral deviation, which gives surgeons more workspace (compared to $33^{\circ}$ and $37.8^{\circ}$ from motion characterization experiments), and gives room to accommodate different techniques and procedures.

\section{E. Design Implementation}

Designing a SFB wrist for microsurgery presents a set of challenges. The performance of a parallel mechanism heavily relies on manufacturing tolerances. Misalignment in assembly can cause excessive loading of joints and actuator couplings, therefore it is crucial to design counter-measures for mitigating these inaccuracies. In addition, the workspace around the patient in the operating room is constrained, therefore it is important for the design to be compact. We chose to implement the design with $W G C I=0.52$, since this design has shorter linkages $\left(\alpha_{1-4}\right)$, meaning that the inertial loading on the actuators are lower.

The base linkage of the SFB is split into two pieces, which allows the mechanism to be assembled easily and leaves room for play between the parts. Two 12-Watt $\varnothing 22 \mathrm{~mm}$ brushless DC motors (EC-max 283840, Maxon, Switzerland) with 53:1 gear reduction are used to actuate the SFB. These two actuators are coupled to the links 1 and 2 of the mechanism via a motor coupling that transmits torques from the motor shaft to the linkage (Fig. 6A). This coupling has two set screws that are tightened on to the motor shaft. The coupling then connects to the linkage via two prongs and two screws. The linkage sits on a ball bearing such that loads on the arm do not cause excessive lateral loading on the actuator output. The passive joints that connect linkages have two high-precision ball bearings, ensuring good axial alignment between linkages.

In addition to the two DoF of the wrist (elevation and lateral deviation), an additional DoF (roll) is attained by

TABLE III

OPTIMIZATION RESULTS.

\begin{tabular}{c|ccccc}
\hline WGCI & $\alpha_{0}$ & $\alpha_{1}$ & $\alpha_{2}$ & $\alpha_{3}$ & $\alpha_{4}$ \\
\hline \hline 0.57 & $45.0^{\circ}$ & $85.0^{\circ}$ & $85.0^{\circ}$ & $76.7^{\circ}$ & $76.7^{\circ}$ \\
\hline 0.52 & $90.0^{\circ}$ & $60.0^{\circ}$ & $60.0^{\circ}$ & $60.0^{\circ}$ & $60.0^{\circ}$ \\
\hline
\end{tabular}

an $\varnothing 8-\mathrm{mm}$ brushless DC motor (EC 384408, Maxon, Switzerland) with 16:1 gear reduction for axially rotating the end-effector. This motor is housed in the output shaft of the SFB (Fig. 6B). There are two ball bearings fitted outside the motor housing that serve to (1) clamp down the motor to prevent it from rotating inside the housing, (2) ensure good axial alignment of the casing with the motor. The casing consists of two halves that are fastened together to clamp onto the two bearings and the motor shaft. The end-effector is attached to the end of this casing.

The linkages and motor couplings were 3D printed. Manufacturing the mechanism using plastic rather than metal helps mitigate the effects of misalignment since the material can slightly deform. However one disadvantage of 3D printing is that the material creeps over time, possibly reducing system accuracy.

Three ball-screw linear stages (ATS100-100, Aerotech, Pittsburgh, PA) were mounted orthogonally to create the 3DoF linear stage. The linear stages have $100 \mathrm{~mm}$ travel with $0.5 \mu \mathrm{m}$ resolution and are connected to a control box (A3200 Npaq Drive Rack, Aerotech, Pittsburgh, PA) that runs an internal servo loop on the stages at $8 \mathrm{kHz}$. Mounted on the linear stage is the SFB wrist. The two $\varnothing 22 \mathrm{~mm}$ Maxon motors are controlled using two digital positioning controllers (EPOS2 24/2, Maxon, Switzerland) that run internal servo loops at $1 \mathrm{kHz}$. The $\varnothing 8 \mathrm{~mm}$ motor is controlled using a servo controller (ESCON 36/3 EC, Maxon, Switzerland).

\section{F. Teleoperation Architecture}

System teleoperation is enabled using a Phantom Omni haptic interface (SensAble Technologies Inc., USA) as the master. A Graphical User Interface (GUI) handles communications with the Phantom Omni, as well as the Aerotech and Maxon motor controllers through provided Application Programming Interface (API) library calls. The GUI was programmed in $\mathrm{C}++$ using the QT application framework (Digia Plc, Helsinki, Finland). A position-based control strategy is used to drive the system. Master-side motions are scaled down on the slave-side, and the amount of scaling can be controlled using the GUI.

The user can switch from position-mode to velocitymode by pushing outwards on the boundary of a virtual sphere, which encloses the position-mode workspace. This teleoperation strategy enables a fine-coarse motion control model, allowing the user to go to a different region of

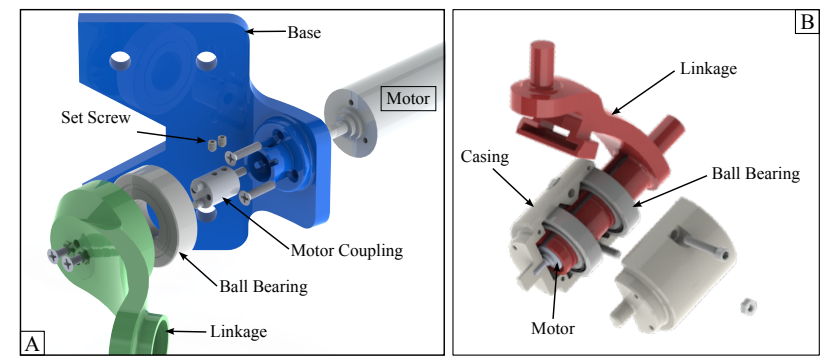

Fig. 6. Detailed views of $(a)$ the actuator-linkage interface, $(b)$ the roll axis motor housing. 


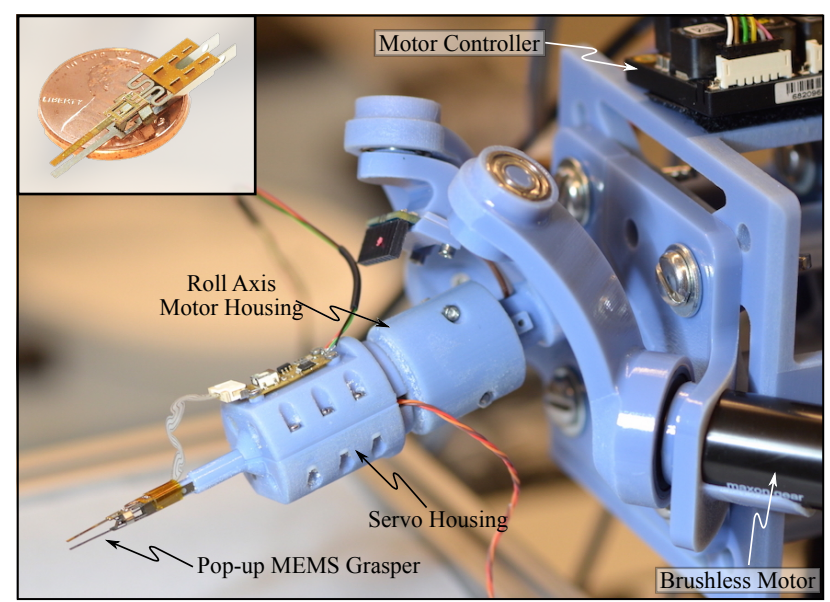

Fig. 7. A picture of the robotic wrist attached to the translation stage, showing actuators, motor controllers, and the pop-up MEMS force sensing surgical grasper. Parts in blue are 3D printed out of VeroBlue material (Stratasys Ltd., USA). Inset shows a close-up view of the grasper [23].

the workspace without having to adjust the motion scaling factors.

\section{SYSTEM CHARACTERIZATION AND VALIDATION}

In order to characterize system behavior, a series of experiments were conducted to measure backlash, bandwidth, step response, and phase shift.

The amount of backlash in the actuated linkages was measured using optical tracking equipment (Claron Inc., Canada). Root mean square (RMS) sensor resolution is $0.25 \mathrm{~mm}$. An optical tracking marker was placed at the passive joint that connects linkages 1 and 3. The linkage was rotated $\mathrm{CW}$ and $\mathrm{CCW}$ by $10.0^{\circ}$ (3000 encoder counts) at $0.033^{\circ}$ (10 e.c.) increments and the change in marker position was measured. After each increment, 10 measurements of the marker position was taken. Data from five consecutive experiments was averaged to reduce variance in readings. Fig. 8 (top) shows a plot of the commanded actuator angle, and the measured linkage position. Analysis shows that the backlash in the system is $1.54^{\circ} \pm 0.2^{\circ}(\mu \pm \sigma)$. This value agrees with the $1.6^{\circ}$ backlash specification provided by the manufacturer for the planetary gearheads (GP 143980, Maxon, Switzerland). This indicates that the backlash in the mechanism is solely due to the backlash in the actuators, and the rest of the design does not introduce any additional backlash. Backlash can be eliminated by using zero-backlash harmonic drives, capstan cable transmissions, or by springloading the linkages. In the case of teleoperation, the human operator can actually correct for backlash easily. [24] shows that backlash only impacts operation time and not accuracy in teleoperation.

System bandwidth was characterized by measuring response to sinusoidal trajectories between $0.5 \mathrm{~Hz}$ and $5 \mathrm{~Hz}$. System response was measured both at the actuator output (by comparing commanded and actual encoder counts), and at the end-effector tip (using optical tracking equipment). Phase delays up to $1^{\circ}$ were observed at the actuator output, with no decay in amplitude (Fig. 8 (bottom)). Optical tracker data was too noisy to determine phase delay, however no decay was observed in amplitude. The actuator response to a $4^{\circ}$ step input was less than $1 \mathrm{~ms}$.

In Fig. 8 (bottom), optical tracker readings indicate that the instrument tip overshoots the target position, however encoder readings from the actuator show no overshoot. The overshoot at the instrument tip may be caused by the flexion of the coupling between the actuator and the linkage, as well as the linkages themselves. These pieces are all 3D printed, and therefore have a lower stiffness compared to that of the actuator components.

Data from the microanastomosis trials show that the angular motion bandwidth of the surgeon is $1.34 \mathrm{~Hz}$. In comparison, the bandwidth of natural hand tremor is 8$12 \mathrm{~Hz}$ [2]. Experiments show that the SFB mechanism has no amplitude decay up to $5 \mathrm{~Hz}$, indicating the mechanism can properly replicate surgeon's hand motions.

In order to demonstrate system capabilities, the micromanipulation system was used in a pick-and-place task, where $1 \mathrm{~mm}$-diameter steel spheres were stacked into a pyramid. A prototype microsurgical gripper based on 'popup MEMS' technology was integrated with the system, and used to manipulate the spheres (Fig. 7). The gripper features a Constantan-based strain gage half-bridge integrated directly into the jaws to enable grip force sensing [23]. On-board signal conditioning, consisting of a Wheatstone bridge and a midpoint-referenced instrumentation amplifier with a gain
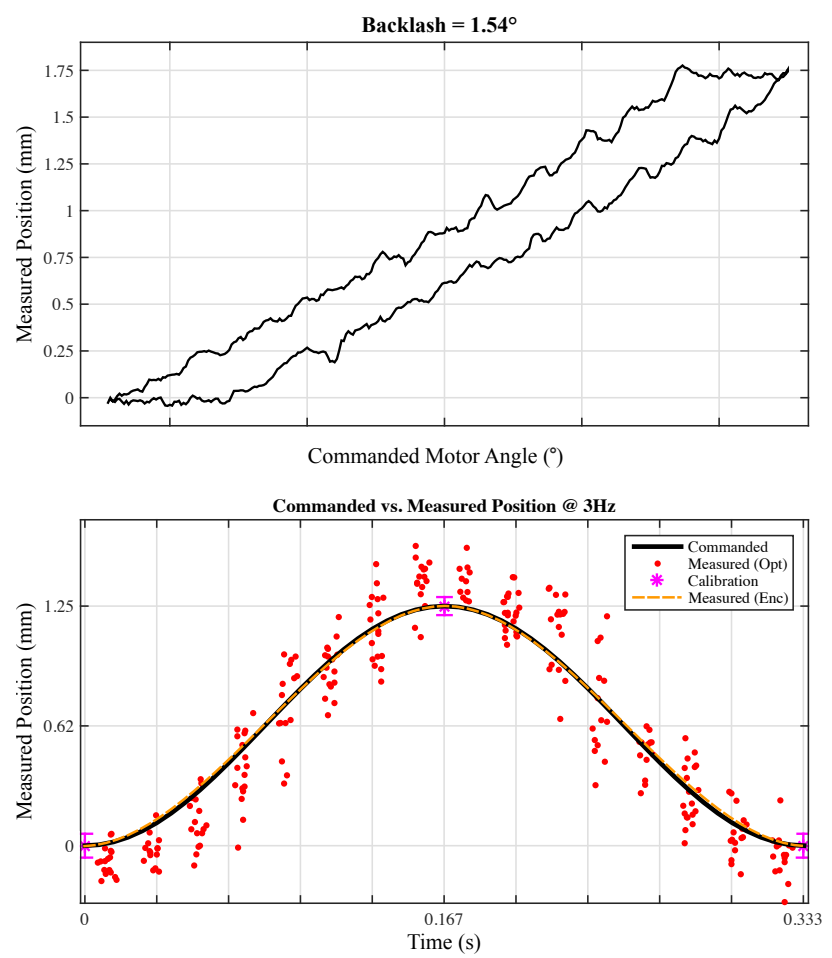

Fig. 8. (top) Hysteresis plot showing measured backlash. (bottom) Sine wave trajectory following. Measured using optical tracking equipment. Black line represents the commanded position. Orange dotted line shows measured position based on encoder readings. Red dots indicate tracker readings at the end-effector. Based on encoder readings, there is $1^{\circ}$ phase delay with no observable dropoff in amplitude. 


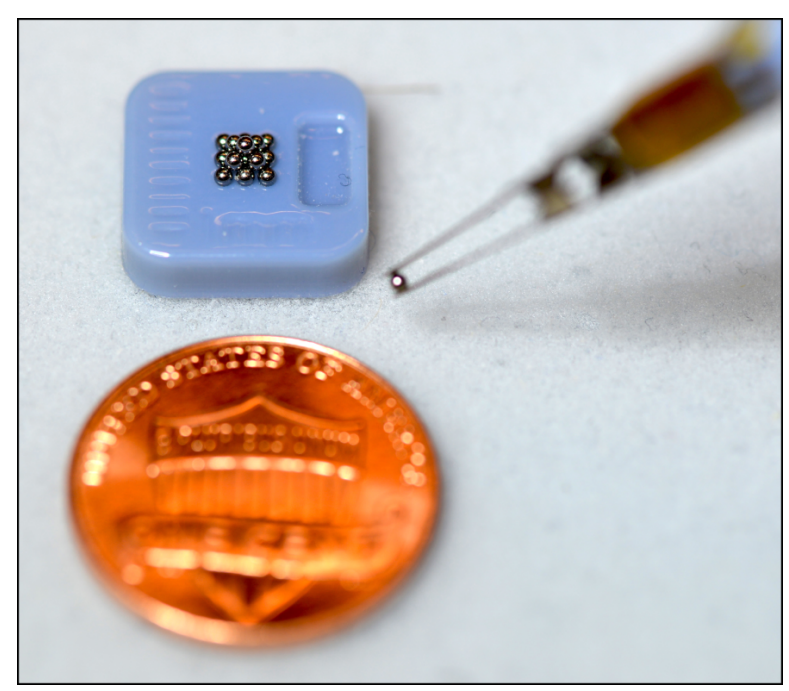

Fig. 9. Final structure after the completion of the pick-and-place task, where 14 steel spheres of $1 \mathrm{~mm}$ diameter were stacked to construct a pyramid. U.S. penny shows scale.

of 1000 , converts the strain measured in the jaws due to an applied force into an analog voltage with a sensitivity of $4.8 \mathrm{~V} / \mathrm{N}$ and a resolution of $1.6 \mathrm{mN}$. A 2.3 gram linear servo (SPMSA2030L, Spektrum, IL, USA) actuates the gripper by pulling on the midpoint of an interior Sarrus linkage which brings the two jaws together in a pinching fashion. During the manipulation task, the gripper measured $32.3 \pm 3.08 \mathrm{mN}$ of grip force required to pick up and displace the metal spheres. Fig. 9 shows the final structure constructed during the experiment. A video of the experiment can be accessed through the following link: https://youtu.be/Mz8XKKpWD1M.

\section{CONCLUSIONS AND FUTURE WORK}

The design, control, and optimization of a new robotic system for microsurgical applications was presented. The kinematic relations for the SFB mechanism were derived. The mechanism design was optimized to maximize workspace and manipulability. The optimized design provides surgeons with the workspace required for performing microsurgical operations. System capabilities were demonstrated in a realtime, teleoperated micromanipulation task.

In future work, calibration algorithms and techniques will be developed to increase the accuracy of the system. The mechanism will be manufactured out of aluminum to increase mechanical rigidity. The use of piezoelectric actuators will be explored to eliminate the backlash in the motors. The grasping forces meaured by the pop-up MEMS grippers were not displayed to user in this study. We are currently designing a haptic interface that can be attached to the Phantom Omni for displaying the gripping forces, allowing the user to feel interaction forces with the environment. New end-effectors will be integrated with the system to allow a variety of tasks to be performed under microscope guidance.

\section{ACKNOWLEDGMENT}

We would like to thank Zhi Ern Teoh for valuable discussions, and Dr. James Weaver for his help with 3D printing.

\section{REFERENCES}

[1] W. Ang, P. Pradeep, and C. Riviere, "Active tremor compensation in microsurgery," in Proc. of 26th Intl. Conf. IEEE Eng. in Med. Bio. Soc. (IEMBS'04), vol. 1, Sept 2004, pp. 2738-2741.

[2] C. Riviere, R. Rader, and P. Khosla, "Characteristics of hand motion of eye surgeons," in Proc. of 19th Intl. Conf. IEEE Eng. in Med. Bio. Soc. (IEMBS'97), vol. 4, Oct 1997, pp. 1690-1693 vol.4.

[3] B. Mitchell, J. Koo, M. Iordachita, P. Kazanzides, A. Kapoor, J. Handa, G. Hager, and R. Taylor, "Development and application of a new steady-hand manipulator for retinal surgery," in 2007 IEEE Intl. Conf. Robotics and Automation (ICRA), April 2007, pp. 623-629.

[4] W. Wei, R. Goldman, N. Simaan, H. Fine, and S. Chang, "Design and theoretical evaluation of micro-surgical manipulators for orbital manipulation and intraocular dexterity," in 2007 IEEE Intl. Conf. Robotics and Automation (ICRA), April 2007, pp. 3389-3395.

[5] R. MacLachlan, B. Becker, J. Cuevas Tabares, G. Podnar, L. Lobes, and C. Riviere, "Micron: An actively stabilized handheld tool for microsurgery," IEEE Trans. Robotics, vol. 28, no. 1, pp. 195-212, Feb 2012.

[6] S. E. Salcudean and J. Yan, "Towards a Force-Reflecting MotionScaling System for Microsurgery," in 1994 IEEE Intl. Conf. Robotics and Automation (ICRA), 1994, pp. 2296-2301.

[7] B. Krapohl, B. Reichert, H. Machens, P. Mailänder, M. Siemionow, and J. Zins, "Computer-guided microsurgery: surgical evaluation of a telerobotic arm.” Microsurgery, vol. 21, no. 1, pp. 22-29, 2001.

[8] R. Li, J. Jensen, and J. Bowersox, "Microvascular anastomoses performed in rats using a microsurgical telemanipulator." Comput Aided Surg., vol. 5, no. 5, pp. 326-332, 2000.

[9] R. D. Katz, G. D. Rosson, J. A. Taylor, and N. K. Singh, "Robotics in microsurgery: Use of a surgical robot to perform a free flap in a pig," Microsurgery, vol. 25, no. 7, pp. 566-569, Jan. 2005.

[10] C. Taleb, E. Nectoux, and P. Liverneaux, "Limb replantation with two robots: a feasibility study in a pig model," Microsurgery, 2009.

[11] J.-L. Bourges, J.-P. Hubschman, B. Burt, M. Culjat, and S. Schwartz, "Robotic microsurgery: corneal transplantation," $\mathrm{Br}$ J Ophthalmol., vol. 93, no. 12, pp. 1672-1675, Dec. 2009.

[12] D. Camarillo, T. Krummel, and J. Salisbury Jr., "Robotic technology in surgery: Past, present, and future," Am. J. Surg., vol. 188, no. 4, pp. 2-15, Oct. 2004.

[13] F. Hammond, S. Talbot, R. Wood, and R. Howe, "Measurement System for the Characterization of Micro-Manipulation Motion and Force," $J$. Med. Devices, vol. 7, no. 3, pp. 030940-1, Sep. 2013.

[14] M. Cutkosky, "On grasp choice, grasp models, and the design of hands for manufacturing tasks," IEEE Trans. Robot. Autom., vol. 5, no. 3, pp. 269-279, Jun 1989.

[15] B. Schena, "Center robotic arm with five-bar spherical linkage for endoscopic camera," U.S. Patent 8469 945, 2013.

[16] B. M. Schena, "Robotic arm with five-bar spherical linkage," U.S. Patent 8506 556, 2013.

[17] M. Lum, J. Rosen, M. Sinanan, and B. Hannaford, "Optimization of a spherical mechanism for a minimally invasive surgical robot: theoretical and experimental approaches," IEEE Trans. on Biomed. Eng., vol. 53, no. 7, pp. 1440-1445, July 2006.

[18] M. Ouerfelli and V. Kumar, "Optimization of a Spherical 5-Bar Parallel Drive Linkage," J. Mech. Des., vol. 116, no. 1, pp. 166-173, Mar. 1994.

[19] C. Gosselin and J. Angeles, "The Optimum Kinematic Design of a Spherical Three-Degree-of-Freedom Parallel Manipulator," J. Mech. Des., vol. 111, pp. 202-207, 1989.

[20] J. P. Merlet, "Jacobian, Manipulability, Condition Number, and Accuracy of Parallel Robots," J. Mech. Des., vol. 128, no. 1, pp. 199-206, Jan. 2006.

[21] L. Birglen, C. Gosselin, N. Pouliot, B. Monsarrat, and T. Laliberte, "Shade, a new 3-dof haptic device," IEEE Trans. Robot. Autom., vol. 18, no. 2, pp. 166-175, Apr 2002.

[22] C. Gosselin and J. Angeles, "A Global Performance Index for the Kinematic Optimization of Robotic Manipulators," J. Mech. Des., vol. 113, no. 3, pp. 220-226, Sep. 1991.

[23] J. Gafford, S. Kesner, R. Wood, and C. Walsh, "Force-sensing surgical grasper enabled by pop-up book mems," in 2013 IEEE/RSJ Intl. Conf. Intelligent Robots and Systems (IROS),, Nov 2013, pp. 2552-2558.

[24] J. Peine, V. Agrawal, and W. Peine, "Effect of backlash on surgical robotic task proficiency," in 2012 4th IEEE Intl. Conf. Biomed. Robotics and Biomech. (BioRob), June 2012, pp. 799-804. 